\title{
Keyword Trends for Mother-Child Oral Health in Korea Based on Social Media Big Data from Naver
}

\author{
Jung-Eun Park ${ }^{1}$, Ja-Won Cho ${ }^{1,2}$, Jong-Hwa Jang ${ }^{1}$ \\ 'Department of Dental Hygiene, College of Health Science, Dankook University, Cheonan, Korea \\ ${ }^{2}$ Department of Preventive Dentistry, College of Dentistry, Dankook University, Cheonan, Korea
}

Objectives: The present study examined trends in search keywords related to the oral health of infants and pregnant women using "social media cafés" on the Korean portal site, Naver. Methods: We obtained data from January 2015 to December 2017, collected by searching for common terms related to oral health, such as "dental caries", "oral health", "scaling", "tooth brushing", and "oral examination". Search results for these terms were organized by frequency and visualized by increase in the font size with increasing frequency. Results: The ranking of keywords on Naver cafés for pregnant women and women with infants was as follows (in descending order): "oral examination", "tooth filling", and "tooth brushing". The "oral health" network was linked to "dental caries", "oral health education", and "tooth brushing". In addition, the analysis of trends of keyword frequencies according to time periods showed that "dental caries" and "oral examination" were of highest interest to the café users. Conclusions: We found a high interest in keywords related to preventive measures for the oral health of infants and children, but there was a lack of awareness regarding the oral health of pregnant women. These findings suggest that prevention in infants and pregnant women is necessary, and that public awareness regarding education about oral healthcare needs to be raised.

Keywords: Infant, Medical Informatics, Oral Health, Pregnant Women, Social Media

\section{Introduction}

The internet usage rate of the Korean population aged 3 years or older was $91.5 \%$ in 2018 , with a $1.2 \%$ increase com-

Submitted: July 1, 2019

Revised: May 15, 2020

Accepted: July 20, 2020

\section{Corresponding Author}

Jong-Hwa Jang

Department of Dental Hygiene, College of Health Science, Dankook University, 119 Dandae-ro, Dongnam-gu, Cheonan 31116, Korea. Tel: +82-41-550-1495, E-mail: jhj@dankook.ac.kr (https://orcid. org/0000-0001-9044-0218)

This is an Open Access article distributed under the terms of the Creative Commons Attribution Non-Commercial License (http://creativecommons.org/licenses/bync/4.0/) which permits unrestricted non-commercial use, distribution, and reproduction in any medium, provided the original work is properly cited.

(c) 2020 The Korean Society of Medical Informatics pared to the previous year. The differences according to age and gender have decreased significantly, indicating that the majority of people are using the internet [1].

Recently, a large volume of unstructured information on social media has been circulating owing to the proliferation and rapid increase in smartphone and internet use. "Social media" includes Internet-based applications that allow people to share their experiences and opinions and exchange comments online [2]. Data based on the social relations of such social media applications are known as "social big data" [3], and research has been conducted in various fields to collect and analyze the large-scale data generated in real-time to derive countermeasures or predict social changes [4]. Moreover, the universality of social media integrates the functions of rapid acquisition of information and personal communication, while it can also be used to identify public interests and trends by focusing on specific issues, social phenomena, 
and keywords [5].

The current study was implemented with the idea that the field of public health and dentistry should identify public interests and trends related to oral health and reflect them in the development of oral health projects and planning.

Regarding the formulation of plans for dental health projects in Korea, projects regarding the dental health of pregnant women and infants must be included and should be managed as dental health projects for mothers and infants as per the Mother and Child Health Act [6].

Pregnant women and infants are thus a target segment of the population and are specifically vulnerable to oral health complications. Parents play an important role in the oral health condition of infants and toddlers [7]. This is particularly true of pregnant women because the oral health condition of the mother is closely related to that of the child [8]. In addition, the oral health behaviors of infants and toddlers affect their entire lives. Therefore, the importance of oral health in infants and pregnant women is emphasized by regulations and various public health services [9].

Because infants and toddlers are susceptible to oral diseases and are unable to care for their own oral health, caregivers are required to help them with oral health management, knowledge acquisition, and habit formation [10]. During this period, the role of parents and caregivers is important and should focus on thorough management and prevention of the dental caries [11,12].

Pregnancy gingivitis in pregnant women is very common [13], and periodontal disease during pregnancy can lead to low birth weight, preeclampsia, and preterm delivery $[14,15]$. Therefore, the oral health of pregnant women and infants is important systemically and must be managed at the clinical and government levels.

The identification of common search keywords and common perceptions regarding oral health using the social media big data mentioned above in mother-and-child subjects may enable us to develop more practical oral health projects and conduct more effective promotional activities. Two examples of social big data analyses are trend analyses of health technologies [16] and network analyses in health informatics [17]. There are also cases in which diseases are predicted by the analysis of search trends [18]. Such analyses of public perceptions are, however, field- and country-specific. So far, there have been no big data analyses in the field of dentistry and dental hygiene in Korea. The existing methods of survey research to understand public perceptions have their limits because it is necessary to analyze social media big data associated with actual public perceptions and not simply base analyses on the oral condition index provided by experts.

In this study, we focused on online child-rearing communities, so-called "mom cafés", where concerns about childrearing are shared and social learning through interaction is carried out. The trends of mom cafés were analyzed and compared with those of other cafés, including those run by dental clinics, to investigate the two main subjects of mother-and-child oral health, and infants and mothers.

Mom cafés are personally run, online child-rearing communities, which have the "café" format provided by Naver. Members are mainly parents of infants and preschool children as well as prospective parents [19]. Mom cafés are characterized by bidirectional communication regarding pregnancy, childbirth, and child-rearing and are subcategorized into various dashboards. Hence, mom cafés are considered to better reflect the search keywords regarding mother-andchild oral health than any other online communities.

This study investigated the trend in the search keywords regarding the oral health of infants and expectant mothers and analyzed social media big data concerning oral health in so-called mom cafés, whole cafés, and dental cafés belonging to a domestic portal (Naver).

\section{Methods}

\section{Data Acquisition and Analysis}

The data were obtained from a café on Naver, a Korean portal site, and keywords from 3 years (from January 2015 to December 2017) were used in the search. The keywords were not technical expressions but daily terms, and the results were obtained by conducting searches using the terms "dental caries", "dental health", "scaling", "tooth brushing", "oral examination", "periodontal disease", "plaque", "oral disease", "sealant", "dental health education", and "fluoride brushing". We also collected information regarding the title, date, and the URL of each café post.

Mom cafés, whole cafés, and dental cafés were analyzed in this study. Mom cafés refers to online child-rearing cafés in Naver, where information about childbirth and child-rearing is shared. Whole cafés refers to all of the Naver online cafés within the period of data collection. Dental cafés refers to the online cafés opened by dental clinics.

Because web pages such as Naver blogs, news sites, and newspaper articles have different structures, we used the Python Selenium library to control the web browser remotely, and a search was conducted every month, as the number of articles appearing on a web page can be up to 1,000 per search. Additionally, a sufficient time interval was given be- 
fore requesting the next web page, to avoid IP blocking and to maintain the internet speed. A total of 179,536 extracted terms were stored in the MySQL database, and the necessary information was extracted for analysis.

\section{Pre-treatment Data and Analysis}

The data extracted for the analysis in this study included the title and date of each post. The morphological analysis was performed using the Python KoNLPy library, and the extracted terms were organized by frequency and visualized using the word cloud method, by increasing the font size with increasing frequency. In addition, the frequency of each keyword was analyzed to confirm the awareness level for each keyword. By performing a network analysis, we determined how the dental health-related keywords were related to each other.

\section{Results}

\section{Ranking and Frequency of Oral Health-Related Keywords} Table 1 show the ranking and frequency of the search keywords. The rankings of all Naver cafés overall were different from those of specific dental cafés and mom cafés. The overall results of all cafés showed the terms "dental caries", "dental health", and "scaling" at the top. However, the search results from the dental and mom cafés were slightly different, with "dental caries", "oral examination", and "scaling" being the most frequently used terms. When further classifying the words in detail, on dental cafés, rankings were high for "periodontal disease" and "dental health", whereas mom cafés showed higher frequencies for "oral examination", "tooth fill- ing", and "tooth brushing", at 59.12\%, 42.79\%, and $31.79 \%$, respectively.

\section{Network Analysis of the Keywords Related to Oral Health} The results of the network analysis using the results shown in the title of the posts from the overall Naver cafés search results on oral health-related keywords are shown in Figure 1. The main keywords that the general public frequently use in the titles of their posts in relation to oral health are "dental caries" and "scaling". The nodes of these two keywords were the largest, and "dental caries" and "scaling" were linked to most keywords, such as "tooth brushing", "oral examination", "periodontal disease", and "fluoride".

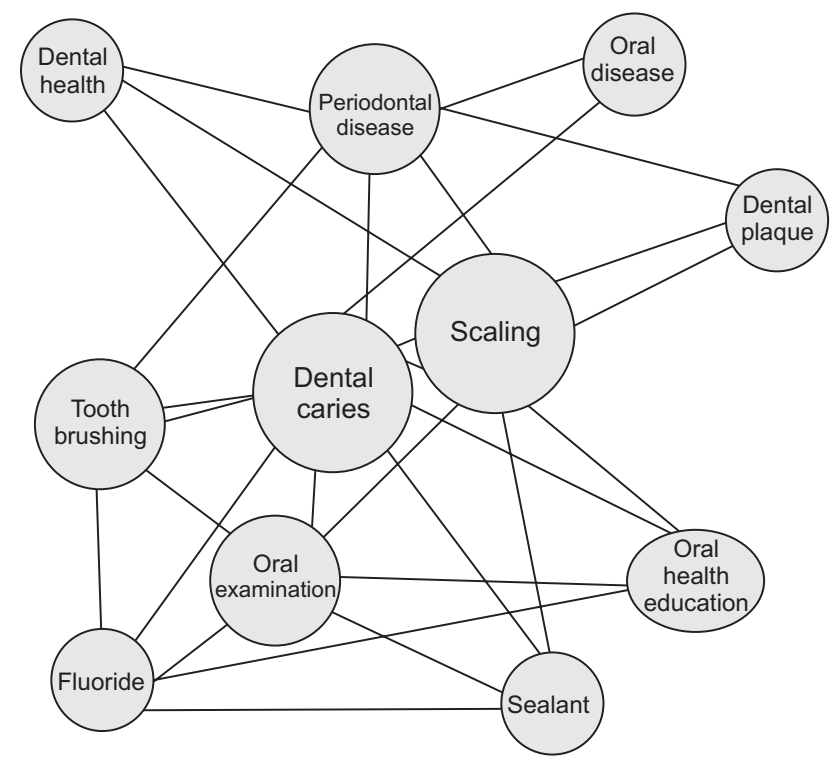

Figure 1. Analysis of the oral health network.

Table 1. Rank and frequency of the search results for oral health-related keywords

\begin{tabular}{clccccc}
\hline \multirow{2}{*}{ Ranking } & \multirow{2}{*}{ Search word } & \multicolumn{2}{c}{ Domestic search results } & \multicolumn{2}{c}{ Overall search results } \\
\cline { 3 - 4 } \cline { 5 - 6 } & & All Naver cafes & Dental and mom cafes & & Café of dental clinic & Café of mom \\
\hline 1 & Dental caries & 92,694 & 23,871 & $4,921(5.31)$ & $18,950(20.44)$ \\
2 & Dental health & 22,631 & 5,950 & $4,076(18.01)$ & $1,874(8.28)$ \\
3 & Scaling & 17,931 & 7,882 & $2,968(16.55)$ & $4,914(27.41)$ \\
4 & Tooth brushing & 16,607 & 7,115 & $1,835(11.05)$ & $5,280(31.79)$ \\
5 & Oral examination & 14,201 & 9,204 & $808(5.69)$ & $8,396(59.12)$ \\
6 & Periodontal disease & 7,191 & 2,359 & $1,443(20.07)$ & $916(12.74)$ \\
7 & Dental plaque & 3,989 & 1,344 & $289(12.23)$ & $627(15.72)$ \\
8 & Oral disease & 2,364 & 494 & $118(10.56)$ & $205(8.67)$ \\
9 & Sealant & 1,117 & 596 & 172 & $478(42.79)$ \\
10 & Oral health education & 471 & 72 & $15(4.41)$ & $116(24.63)$ \\
\hline
\end{tabular}

Values are presented as frequency (\%). 


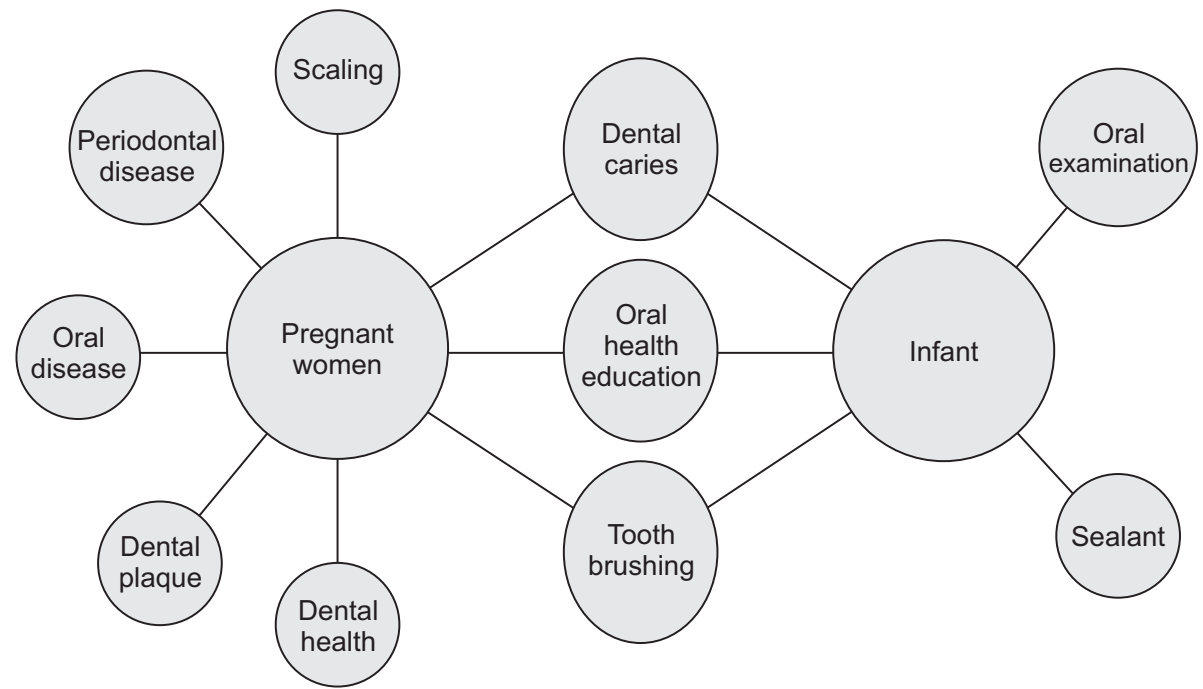

Figure 2. Analysis of the oral healthrelated network for infants and pregnant women.

\section{Network Analysis of the Keywords Related to Oral}

\section{Health in Infants and Pregnant Women}

The results of our analysis of the network among the keywords of oral health for infants and pregnant women are shown in Figure 2. The keywords associated with pregnant women were "scaling", "periodontal disease", "oral disease", "plaque", and "dental health", whereas those linked to infants and toddlers were "oral examination" and "sealant" as well as "dental caries", "oral health education", and "tooth brushing".

\section{Trends in Frequency Change by Period and Word Cloud Analysis}

The change in frequency by period was identified for the top five search results, and they included "dental caries", "dental health", "scaling", "tooth brushing", and "oral examination". The results of the word cloud analysis using the top 200 morphemes are shown in Figure 3.

Figure $3 \mathrm{~A}$ is a graph showing the frequency of the search results for the keyword "dental caries" by period. In March of every year, interest in "dental caries" increased sharply in all cafes, and the frequency gradually increased with the number of months. In addition, word cloud analysis showed that there was a high interest in "cavity treatment" for children. The most relevant keywords for "dental caries" were "dental clinic", "children", "scaling", "root canals", and "dental insurance".

Figure $3 \mathrm{~B}$ is a graphical representation of the frequency of the search results for "dental health" by period. Except for the fall of 2017, there seems to have been a growing interest in dental health as a whole. Word cloud analysis shows that "implants", "orthodontics", and "dentistry" are also prominent.

Figure 3C is for the keyword "scaling", and the interest of the public rose sharply in June of 2017 due to the change in the insurance coverage period for scaling. In addition, interest in scaling in mom cafes gradually increased. Word cloud analysis showed that the keyword with high relevance to scaling was "health insurance".

Figure 3D is for the keyword "tooth brushing", indicating that the public's interest in tooth brushing was steadily increasing. In March, April, June, and July of 2015, there was a sharp increase in interest, but the keyword "tooth brushing" in dental cafes appeared significantly less frequently compared to other communities. Word cloud analysis showed that "toothpaste", "dental clinic", "children", "implant", and "scaling" were the most relevant keywords for tooth brushing.

Figure 3E is for the keyword "oral examination". In comparison to other search keywords, the interest in mom cafes was very high for "oral examination", and the keywords with high relevance were "infant and toddler" and "oral examination".

\section{Discussion}

The social network services (SNSs) mainly used in Korea in 2017 were Facebook, Kakao Story, and Instagram, while Naver cafés belonging to the "community" category of social media accounted for $10.7 \%$ of SNS use [1].

Among these, mom cafés are an internet space where users who are interested in topics related to pregnant women, parents of infants and toddlers, and children come together grouped by region and interests, to form a community and share information. Therefore, we decided that using these mom cafés in community social media could enable us to understand the interests and perceptions of pregnant women 


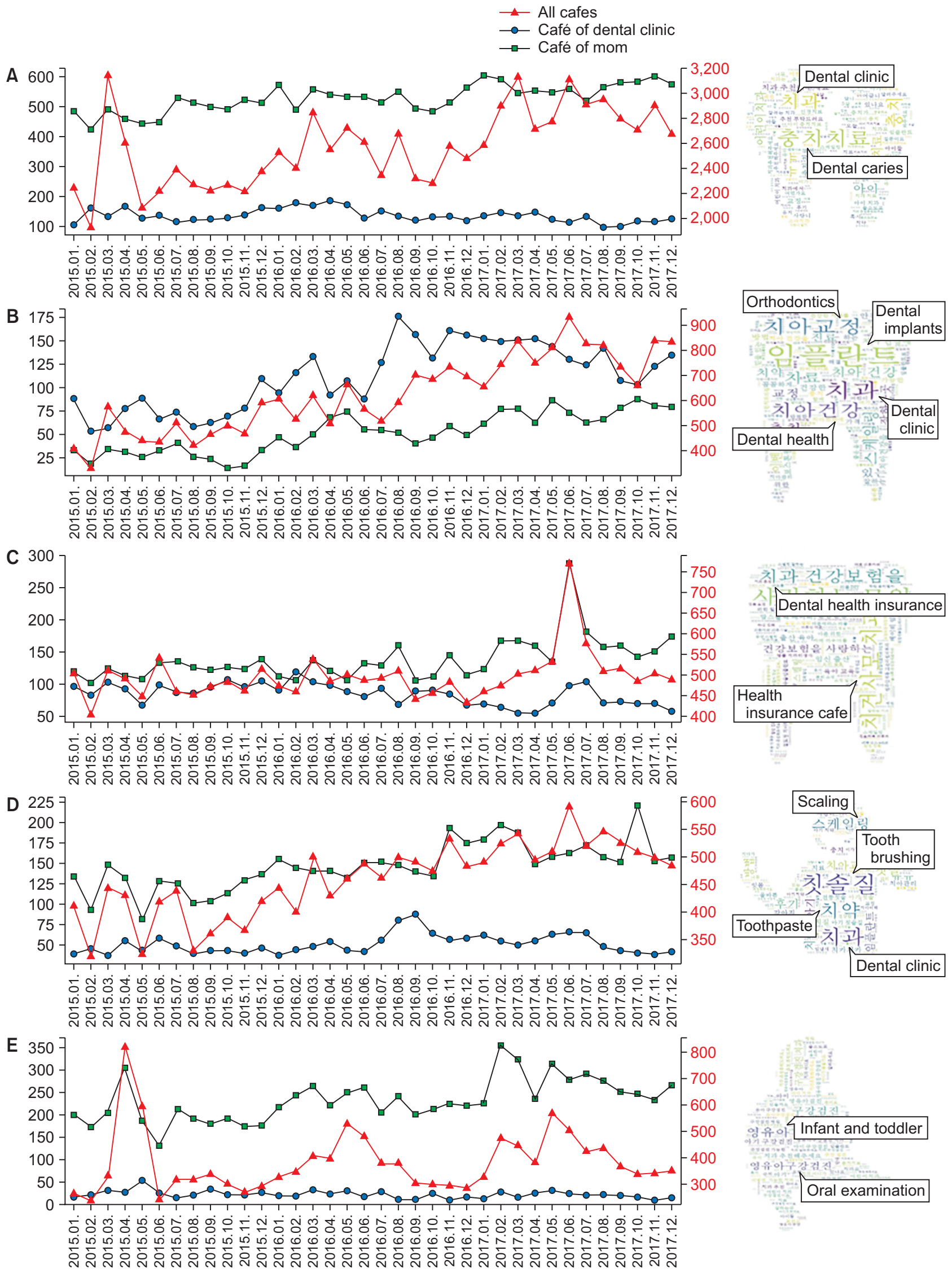

Figure 3. Graph and word cloud for the frequency of the search results for each keyword by period: (A) dental caries, (B) dental health, (C) scaling, (D) tooth brushing, and (E) oral examination. 
and women with infants more effectively.

First, to confirm the general public's awareness of and interest in dental health, the frequencies of search results from all cafés, dental clinic/hospital cafés, and mom cafés were examined. Our results showed that the rankings for each keyword differed according to the type of café. The top search keywords for the mom cafés mainly targeting pregnant women appeared to be "oral examination", "sealant", and "tooth brushing".

Dental examinations are preventive activities for the early detection and treatment of oral diseases, and they are administered three times from 18 to 65 months of age in infants and toddlers [20]. Children who have undergone oral examinations later need less medical care than do those who have not, demonstrating the effectiveness of early examinations in reducing pain and medical costs [21]. Our data confirm that awareness of and interest in oral examinations is greater among users of mom cafés compared to users of other cafés.

Dental sealants were first included in the National Health Insurance (NHI) benefits in December 2009 for the first molar in children aged 6-14 years and for the second molar in October 2012. The qualifying age for the benefit has since been continuously expanded to include anyone under the age of 18 years in May 2013 [22]. In addition to oral health examinations for infants and toddlers, dental sealants are used a preventive measure for dental caries in children, and they are of high interest to parents who have young children because they are covered by the NHI. Our findings confirm that parents were actively sharing information on the top three items, namely, "oral examination", "sealant", and "tooth brushing" on mom cafés because these are essential for dental care in infants and toddlers.

On the other hand, "periodontal disease" was the most frequent result for keywords used in dental clinic/hospital cafés. Generally, social media sites operated by clinics/hospitals are for specific patients, and their interests differ from those for pregnant women and women with infants. This indicates that the keywords of interest regarding oral health in the general public vary depending on the specific subject and the nature of the community.

Medical consumers receive information about doctors, clinics, and healthcare through social networking web sites, such as blogs run by doctors, online communities, or YouTube, as well as specialized health portal sites. In addition, SNS information provided by medical institutions is regarded as a verified resource, and it influences the formation of a positive and trustworthy image [23].
From 2008 to 2018, the prevalence of periodontal disease in the Korean population aged 19 years or older decreased by $8.7 \%$ [24]. However, periodontal disease is the main cause of missing teeth and major oral disease; therefore, thorough oral healthcare is necessary. Accordingly, the search keywords regarding adult oral diseases are ranked at the top in dental cafés.

The results of the analysis of the network of oral healthrelated keywords for infants and pregnant women showed that the network consisted of keywords related to prevention in infants and toddlers, while those for pregnant women included "periodontal disease" and "oral disease". As previously mentioned, there was great interest in the preventive treatment and management of infants and toddlers because this is essential for oral care, but "prevention" in pregnant women seemed to be of interest to only a minority, and very little interest was observed for "scaling".

Oral health education and oral examinations are provided for pregnant women, and Mother and Child Health Pocketbooks are issued to those with infants, according to the Mother and Child Health Act [6]. Various services such as fluoride coating and tooth brushing education are also being provided by community health centers. In particular, it is highly important to implement prevention activities, such as oral health education, for pregnant women. It has been reported that a mother's oral health knowledge has a greater influence on the oral healthcare of the child than the father's because the mother spends more time with the child [25].

It has also been reported that the child's dental caries experience is directly proportional to the mother's dental caries experience [26]. Therefore, children's level of oral health may differ according to their mothers' oral health knowledge and behavior.

This shows that it is necessary to promote awareness and change the perception of oral health examinations, oral health education, and preventive management in pregnant women.

We also analyzed trends in changes in the frequency of the search terms "dental caries", "dental health", "scaling", "tooth brushing", and "oral examination" according to the search period. We found that the frequency of all keywords increased overall in 2016 and 2017 compared to January 2015. This may be a result of the increased use of the internet, but it also suggests that public awareness of oral health increased.

The frequency of the keyword "dental caries" increased over time on all cafés, especially on the mom cafés. In addition, the word cloud related to dental caries was focused on 
the treatment of the affected teeth rather than on preventive treatment. Dental caries in infants is an infectious and contagious disease, which affects the surfaces of multiple teeth simultaneously, and its incidence rapidly increases until childhood [27]. According to a survey of the oral health status of children in Korea conducted in 2015, the percentage of childhood dental caries was $62.2 \%$ in 2012 and $64.4 \%$ in 2015 , suggesting that preventative activities related to dental caries should be actively promoted $[28,29]$.

On the other hand, the search term "dental health" was characterized by a significantly higher frequency on all cafés and on dental cafés, compared to mom cafés, while "implants" and "orthodontics" were prominent terms in the word cloud. Implants and orthodontic treatment are tertiary preventative dental treatments that are applied for oral rehabilitation in cases where tooth loss and malocclusion are evident. Therefore, the keyword "dental health" in the public seems to be perceived as treatment-oriented rather than prevention-oriented. The keyword "scaling" showed a sudden increase in frequency on mom cafés and across all cafés in June 2017. This suggests that the frequency of the keyword search increased due to a change in the coverage period for scaling implemented around that time. The keywords "tooth brushing" and "oral examination" were dominant on mom cafés, while their frequencies were very low across cafés of general dental clinics/hospitals, indicating a difference in interests.

To summarize, the present study analyzed dental healthrelated keywords used on community SNSs that are used mainly by mothers, in comparison to those used by the general public. We found a difference between the interests of the general public and those expressed on the mom cafés. In addition, there was an interest in preventive management, such as oral examinations, dental sealants, and tooth brushing to improve oral health in infants and young children, but it seems there is a need to raise awareness of prevention management among pregnant women. Furthermore, as the perception of dental caries is still focused on treatment rather than prevention, it is necessary to change this perception and provide oral health education for pregnant women and women with infants as well as for the general public. This study was conducted using a single portal site at one timepoint; therefore, the results cannot be generalized to all pregnant women and women with infants. The perceptions of the general population need to be further assessed through the analysis of big data obtained from a variety of subject populations.

\section{Conflict of Interest}

No potential conflict of interest relevant to this article was reported.

\section{Acknowledgments}

The present study was carried out with the support of the 2018 Civilian Routine Support Project from the Ministry of Health and Welfare.

\section{ORCID}

Jung-Eun Park (https://orcid.org/0000-0002-5897-5594)

Ja-Won Cho (https://orcid.org/0000-0003-1458-0416)

Jong-Hwa Jang (https://orcid.org/0000-0001-9044-0218)

\section{References}

1. Korea Internet \& Security Agency. 2018 Survey on the Internet usage [Internet]. Seoul, Korea: Korea Internet \& Security Agency; 2018 [cited at 2020 May 10]. Available from: https://www.kisa.or.kr/public/library/etc_View.jsp ?regno $=0012002 \&$ searchType $=\&$ searchKeyword $=\&$ page Index $=3$.

2. Lee BH. A study on the determinant factors of the social capital construction through social media: Focused on college students. J Internet Comput Serv 2016;17(2):97107.

3. Park CL, Lim ST, Cha SY, Moon JH, Lee IS, Kim JW. A qualitative study on social relationship among social media users: focusing on the weak-tie formation of social network theory. Proceedings of the HCI Society of Korea Conference (HCI2009); 2009 Feb 9-13; Gangwon, Korea. p. 1321-9.

4. Chang SB, Sung YH. Online opinion analysis of performing arts through social big data. J Korea Des Forum 2017;57:79-90.

5. Yun E, Park Y. Public perception and usage pattern of science museum by social media big data analysis. J Korean Assoc Sci Educ 2017;37(6):1005-14.

6. Korea Law Information Center. Dental Health Act [Internet]. Sejong, Korea: Korea Law Information Center; c2012 [cited at 2020 May 24]. Available from: http://www.law.go.kr/lsInfoP.do?lsiSeq=208458\&ef $\mathrm{Yd}=20191024 \# 0000$.

7. Kim GH, Lee KH. Perception of smartphone applications for oral health care education in infants and tod- 
dlers. J Korean Soc Dent Hyg 2018;18(6):987-1001.

8. Kang YM, Cho YS. Impact of mother's oral health literacy on preschool children's oral health status and behavior. J Dent Hyg Sci 2016;16(1):26-36.

9. Ahn YS, Kim MJ. A epidemiological study on the oral health in preschool children for the development of community based oral health program in Sungnam city. J Korean Soc Dent Hyg 2001;1(2):201-11.

10. Lee SN, Kim ES. Comparison between early childhood teachers and mothers in perception of oral health behavior and education for children. J Dent Hyg Sci 2013;13(2):125-34.

11. Lee YM, Yeun YR, Kim SJ. Relation with mothers of oral health knowledge and practice for infants oral health management. J Korea Acad-Ind Coop Soc 2016;17(3): 407-13.

12. Lee SN, Lim SR. In-depth interview of parents experienced first infant oral examination. J Dent Hyg Sci 2017;17(6):543-51.

13. Barak S, Oettinger-Barak O, Oettinger M, Machtei EE, Peled M, Ohel G. Common oral manifestations during pregnancy: a review. Obstet Gynecol Surv 2003;58(9): 624-8.

14. Ryalat S, Sawair F, Baqain Z, Barghout N, Amin W, Badran $D$, et al. Effect of oral diseases on mothers giving birth to preterm infants. Med Princ Pract 2011;20(6): 556-61.

15. Laine MA. Effect of pregnancy on periodontal and dental health. Acta Odontol Scand 2002;60(5):257-64.

16. Lee J, Kim J, Hong YJ, Piao M, Byun A, Song H, et al. Health information technology trends in social media: using twitter data. Healthc Inform Res 2019;25(2):99105.

17. Saheb T, Saheb M. Analyzing and visualizing knowledge structures of health informatics from 1974 to 2018: a bibliometric and social network analysis. Healthc Inform Res 2019;25(2):61-72.

18. Verma M, Kishore K, Kumar M, Sondh AR, Aggarwal G, Kathirvel S. Google Search Trends predicting disease outbreaks: an analysis from India. Healthc Inform Res 2018;24(4):300-8.

19. Kim M, Kim H. Participation of mothers in the internet community: Relationship to their perceptions of social support and parenting efficacy. Korean J Child Stud 2007;28(3):1-17.

20. Ministry of Health and Welfare. Oral examination [In- ternet]. Sejong, Korea: Ministry of Health and Welfare; c2019 [cited at 2020 May 24]. Available from: http:// www.mohw.go.kr/react/policy/index.jsp?PAR_MENU_ ID $=06 \&$ MENU_ID $=06330202 \&$ PAGE $=2 \&$ top Title $=$.

21. Ahn E, Shin H. Effectiveness of oral examination for infants and toddlers: effects on subsequent utilization and costs. J Korean Acad Oral Health 2017;41(2):73-9.

22. Lee $\mathrm{HJ}, \mathrm{Bae} \mathrm{KH}$. A change in the regional disparity based on the national insurance coverage of dental sealant in Korea. J Korean Acad Oral Health 2014;8(3):1659.

23. Sagong M, Jung SH, Park JS, Cho KW. The effects of SNS information characteristics on hospital selection and satisfaction degree. Korean J Health Serv Manag 2014;8(1):15-25.

24. Korea Centers for Disease Control and Prevention. Noncommunicable disease (NCD) statistics [Internet]. Cheongju, Korea: Korea Center for Disease Control and Prevention; c2020 [cited at 2020 May 10]. Available from: https://www.cdc.go.kr/board/board.es? $\mathrm{mid}=\mathrm{a} 206$ 02010000\&bid=0034\&list_no $=366370 \&$ act $=$ view.

25. Kong MS, Lee HS, Kim SN. Children's dental health behavior in relation to their mothers' dental health knowledge level, attitude toward dentist and dental health behaviors. J Korean Acad Dent Health 1994;18(1):84-94.

26. Maciel SM, Marcenes W, Watt RG, Sheiham A. The relationship between sweetness preference and dental caries in mother/child pairs from Maringa-Pr, Brazil. Int Dent J 2001;51:83-8.

27. Caufield PW, Cutter GR, Dasanayake AP. Initial acquisition of mutans streptococci by infants: evidence for a discrete window of infectivity. J Dent Res 1993;72(1):3745.

28. Korean Statistical Information Service. Korean chidren's oral health survey [Internet]. Sejong, Korea: Korean Statistical Information Service; 2015 [cited at 2020 May 24]. Available from: kosis.kr/statHtml/statHtml. do? orgId=117\&tblId=DT_11751N_186\&vw_cd=MT_ ZTITLE\&list_id=117_11751_H_A02\&seqNo=\&lang $\operatorname{mode}=$ ko\&language $=$ kor $\&$ obj_var_id $=\& i t$ m $_{-}$ $\mathrm{id}=\&$ conn_path=MT_ZTITLE.

29. Yeo AN, Kang YM, Lee SY. Factors influencing unmet dental needs of preschool children: a study based on data of the 2013-2015 Korea National Health and Nutrition Examination Survey (KNHNES). J Korean Soc Dent Hyg 2019;19(1):117-29. 